\title{
Recursive Graphical Construction for Feynman Diagrams and Their Weights in Ginzburg-Landau Theory
}

\author{
H. Kleinert, A. Pelster \\ Institut für Theoretische Physik, Arnimallee 14 D-14195 Berlin, Germany \\ E-mails: kleinert@physik.fu-berlin.de,pelster@physik.fu-berlin.de \\ B. Van den Bossche \\ Physique Nucléaire Théorique, B5, Université de Liège Sart-Tilman, 4000 Liège, Belgium \\ E-mail: bvandenbossche@ulg.ac.be \\ and \\ Institut für Theoretische Physik, Arnimallee 14 D-14195 Berlin, Germany \\ E-mail: bossche@physik.fu-berlin.de
}

\begin{abstract}
The free energy of the Ginzburg-Landau theory satisfies a nonlinear functional differential equation which is turned into a recursion relation. The latter is solved graphically order by order in the loop expansion to find all connected vacuum diagrams, and their corresponding weights. In this way we determine the connected vacuum diagrams and their weights up to four loops.
\end{abstract}

\section{INTRODUCTION}

Recently, two of us (H.K. and B.V.d.B.) have determined the two-loop effective potential of $O(N)$-symmetric scalar quantum electrodynamics in $4-\epsilon$ dimensions and its $\epsilon$-expansion [1]. Going to higher loop orders requires the calculation of many complicated Feynman integrals associated with vacuum diagrams. In this paper, we show how to find all these diagrams and their weights with the help of a simple recursive technique described in detail in Refs. [2 6 . The basics of the method were formulated in Refs. [7, 8 . For simplicity, the present work is restricted to the theory of a single complex scalar field coupled to electromagnetism, known as Ginzburg-Landau theory or as scalar quantum electrodynamics. In $D=3$ dimensions, this describes the physics of superconductors. Without electromagnetism, we recover the physics of superfluid helium. Here we shall restrict ourselves to the symmetric phase, where the $O(N)$ symmetry is unbroken, which describes the system above the critical temperature $T_{c}$. The more general situation will be dealt with elsewhere.

For a complex boson field, propagators are represented by oriented lines, as in ordinary quantum electrodynamics [4]. When comparing the two expansions we must, however, drop a minus sign for each fermion loop. In addition, the Ginzburg-Landau theory contains four-point functions of the scalar field which are in principle treated in $\phi^{4}$-theory [3]. However, since we deal here with complex fields, the weight of the corresponding graphs cannot be taken from Ref. [3]. There is furthermore a completely new vertex in scalar QED: the quartic seagull coupling between two photon and two scalar fields. By a replacement of the photon lines by scalar lines, they become equivalent to the diagrams coming from the $\phi^{4}$-vertex, apart, of course, from different weights.

The paper is organized as follows. In Section II we define more precisely the theory to be studied. In Section III we introduce basic functional derivatives which allow in Section $[\mathrm{IV}$ to derive a functional differential equation for the free energy. From this follows a recursion relation which allows to find all vacuum diagrams of a given order $L$ from those of the previous orders. This equation is formulated graphically in Section $\mathrm{V}$, where we also determine the vacuum diagrams and the corresponding weights up to four loops.

\section{GINZBURG-LANDAU THEORY}

The generalized Ginzburg-Landau theory deals with a self-interacting complex scalar field coupled minimally to an electromagnetic vector potential. The theory contains two coupling constants $g$ and $e$. The field expectation value $\langle\phi\rangle$ vanishes above $T_{c}$ and the theory has three types of vertices.

The physics can be extracted from the partition function

$$
Z=\int \mathcal{D} \phi \mathcal{D} \phi^{\dagger} \mathcal{D} A \exp (-E)
$$

with thermal fluctuations being governed by the energy functional 


$$
E\left(\phi^{\dagger}, \phi, A_{\mu}\right)=\phi_{1}^{\dagger} G_{12}^{-1} \phi_{2}+\frac{g}{4} V_{1234} \phi_{1}^{\dagger} \phi_{2} \phi_{3}^{\dagger} \phi_{4}+\frac{1}{2} A_{\overline{1}} D_{\overline{12}}^{-1} A_{\overline{2}}+e H_{\overline{1} 12} A_{\overline{1}} \phi_{1}^{\dagger} \phi_{2}+\frac{e^{2}}{2} F_{\overline{12} 12} A_{\overline{1}} A_{\overline{2}} \phi_{1}^{\dagger} \phi_{2} .
$$

Here and below, overlined indices are used for the electromagnetic field. We work in a covariant gauge and assume the photon propagator to have the appropriate form (see Ref. 代). Using the same notation as in Refs. [3, 4 , we keep all equations as compact as possible by assuming Einstein's summation convention not only for the internal degrees of freedom, but also for the space-time indices, for which repeated indices imply an overall integral sign. In this notation the functional matrices in the energy functional (2) have the following symmetries:

$$
\begin{aligned}
D_{\overline{12}}^{-1} & =D_{\overline{21}}^{-1}, \\
V_{1234} & =V_{3214}=V_{1432}=V_{3412}, \\
F_{\overline{12} 12} & =F_{\overline{21} 12} .
\end{aligned}
$$

\section{BASIC FUNCTIONAL DERIVATIVES}

The recursion relation will be derived by performing functional differentiations of the partition function with respect to the propagators and their inverse as well as the vertices. The basic properties of these derivatives are shown in the following equations:

\section{Scalar sector}

$$
\begin{aligned}
\frac{\delta G_{12}^{-1}}{\delta G_{34}^{-1}} & =\delta_{13} \delta_{24}, \\
\frac{\delta G_{12}}{\delta G_{34}} & =\delta_{13} \delta_{24}, \\
\frac{\delta G_{12}}{\delta G_{34}^{-1}} & =-G_{13} G_{42}, \\
\frac{\delta}{\delta G_{34}^{-1}} & =-G_{13} G_{42} \frac{\delta}{\delta G_{12}} .
\end{aligned}
$$

\section{Photon sector}

$$
\begin{aligned}
\frac{\delta D_{\overline{12}}^{-1}}{\delta D_{\overline{34}}^{-1}} & =\frac{1}{2}\left(\delta_{\overline{13}} \delta_{\overline{42}}+\delta_{\overline{14}} \delta_{\overline{32}}\right), \\
\frac{\delta D_{\overline{12}}}{\delta D_{\overline{34}}} & =\frac{1}{2}\left(\delta_{\overline{13}} \delta_{\overline{42}}+\delta_{\overline{14}} \delta_{\overline{32}}\right), \\
\frac{\delta D_{\overline{12}}}{\delta D_{\overline{34}}^{-1}} & =-\frac{1}{2}\left(D_{\overline{13}} D_{\overline{42}}+D_{\overline{14}} D_{\overline{32}}\right), \\
\frac{\delta}{\delta D_{\overline{34}}^{-1}} & =-D_{\overline{13}} D_{\overline{42}} \frac{\delta}{\delta D_{\overline{12}}} .
\end{aligned}
$$

\section{Vertex derivatives}

$$
\begin{aligned}
& \frac{\delta H_{\overline{1} 12}}{\delta H_{\overline{2} 34}}=\delta_{\overline{12}} \delta_{13} \delta_{24}, \\
& \frac{\delta V_{1234}}{\delta V_{5678}}=\frac{1}{4}\left(\delta_{15} \delta_{26} \delta_{37} \delta_{48}+\delta_{17} \delta_{26} \delta_{35} \delta_{48}+\delta_{15} \delta_{28} \delta_{37} \delta_{46}+\delta_{17} \delta_{28} \delta_{35} \delta_{46}\right), \\
& \frac{\delta F_{\overline{12} 12}}{\delta F_{\overline{34} 34}}=\frac{1}{2}\left(\delta_{\overline{13}} \delta_{\overline{24}} \delta_{13} \delta_{24}+\delta_{\overline{14}} \delta_{\overline{23}} \delta_{13} \delta_{24}\right) .
\end{aligned}
$$


This is a direct extension of relations used in Refs. [3] 6 . The main difference comes from the fact that the previous scalar propagators were symmetric in the indices whereas the present propagators describing complex fields are not. By the chain rule of differentiations, we can then find the derivative of any functional with respect to the propagators and their inverse as well as the vertices.

\section{FUNCTIONAL DIFFERENTIAL EQUATION FOR FREE ENERGY}

With the definitions given in the previous section, we are now prepared to derive the graphical recursion relation for the vacuum graphs. We start from the identity

$$
\int \mathcal{D} \phi \mathcal{D} \phi^{\dagger} \mathcal{D} A \frac{\delta}{\delta \phi_{1}^{\dagger}}\left[\phi_{2}^{\dagger} \exp (-E)\right]=0
$$

and obtain

$$
\int \mathcal{D} \phi \mathcal{D} \phi^{\dagger} \mathcal{D} A\left(\delta_{12}-\phi_{2}^{\dagger} \frac{\delta E}{\delta \phi_{1}^{\dagger}}\right) \exp (-E)=0
$$

which, using the Ginzburg-Landau energy functional (2), leads to the linear equation

$$
\int \mathcal{D} \phi \mathcal{D} \phi^{\dagger} \mathcal{D} A\left(\delta_{12}-\phi_{2}^{\dagger} G_{13}^{-1} \phi_{3}-\frac{g}{2} V_{1345} \phi_{2}^{\dagger} \phi_{3} \phi_{4}^{\dagger} \phi_{5}-\frac{e^{2}}{2} F_{\overline{12} 13} A_{\overline{1}} A_{\overline{2}} \phi_{2}^{\dagger} \phi_{3}-e H_{\overline{1} 13} A_{\overline{1}} \phi_{2}^{\dagger} \phi_{3}\right) \exp (-E)=0
$$

Rewritten in terms of functional derivatives with respect to the inverse of the propagators, this becomes

$$
\int \mathcal{D} \phi \mathcal{D} \phi^{\dagger} \mathcal{D} A\left(\delta_{12}+G_{13}^{-1} \frac{\delta}{\delta G_{23}^{-1}}-\frac{g}{2} V_{1345} \frac{\delta}{\delta G_{23}^{-1}} \frac{\delta}{\delta G_{45}^{-1}}-e^{2} F_{\overline{12} 13} \frac{\delta}{\delta D_{\overline{12}}^{-1}} \frac{\delta}{\delta G_{23}^{-1}}+e H_{\overline{1} 13} A_{\overline{1}} \frac{\delta}{\delta G_{23}^{-1}}\right) \exp (-E)=0
$$

The last part of the above equation may be replaced by a three-vertex derivative. However, proceeding in this way would not lead to an iterative generation of diagrams. For this, it is necessary to consider a second identity:

$$
\int \mathcal{D} \phi \mathcal{D} \phi^{\dagger} \mathcal{D} A \frac{\delta}{\delta A_{\overline{1}}} \exp (-E)=0
$$

from which we obtain

$$
\int \mathcal{D} \phi \mathcal{D} \phi^{\dagger} \mathcal{D} A\left(D_{\overline{12}}^{-1} A_{\overline{2}}+e H_{\overline{1} 12} \phi_{1}^{\dagger} \phi_{2}+e^{2} F_{\overline{12} 12} A_{\overline{2}} \phi_{1}^{\dagger} \phi_{2}\right) \exp (-E)=0
$$

or, equivalently,

$$
\int \mathcal{D} \phi \mathcal{D} \phi^{\dagger} \mathcal{D} A\left(D_{\overline{12}}^{-1} A_{\overline{2}}-e H_{\overline{1} 12} \frac{\delta}{\delta G_{12}^{-1}}-e F_{\overline{12}} 12 \frac{\delta}{\delta H_{\overline{2} 12}}\right) \exp (-E)=0
$$

Inserting the photon-field expectation value (23) into (20), we obtain

$$
\begin{aligned}
\left(\delta_{12}+G_{13}^{-1} \frac{\delta}{\delta G_{23}^{-1}}-\frac{g}{2} V_{1345} \frac{\delta}{\delta G_{23}^{-1}} \frac{\delta}{\delta G_{45}^{-1}}-e^{2} F_{\overline{12} 13} \frac{\delta}{\delta D_{\overline{12}}^{-1}} \frac{\delta}{\delta G_{23}^{-1}}\right. \\
\left.\quad+e^{2} H_{\overline{1} 13} D_{\overline{12}} H_{\overline{2} 45} \frac{\delta}{\delta G_{23}^{-1}} \frac{\delta}{\delta G_{45}^{-1}}+e^{2} H_{\overline{1} 13} D_{\overline{12}} F_{\overline{23} 45} \frac{\delta}{\delta G_{23}^{-1}} \frac{\delta}{\delta H_{\overline{3} 45}}\right) Z=0 .
\end{aligned}
$$

This equation is linear, but it leads to a huge number of diagrams, both connected and disconnected. We remove the disconnected ones by introducing the free energy $W$ as generating functional of the connected Green functions as

$$
Z \equiv \exp (W), \quad W=W_{0}+W_{\text {int }},
$$

where $W_{0}$ is the free field part 


$$
W_{0}=-\operatorname{Tr} \ln \mathrm{G}^{-1}-\frac{1}{2} \operatorname{Tr} \ln \mathrm{D}^{-1},
$$

with $\operatorname{Tr}$ being a shorthand notation for the functional trace. Working with the series representation of the logarithm, we obtain directly the relations

$$
\begin{aligned}
& \frac{\delta W_{0}}{\delta G_{12}^{-1}}=-G_{21}, \\
& \frac{\delta W_{0}}{\delta D_{\overline{12}}^{-1}}=-\frac{1}{2} D_{\overline{21}}, \\
& \frac{\delta W_{0}}{\delta H_{\overline{1} 23}}=0,
\end{aligned}
$$

where the index ordering is important for the complex scalar fields.

Introducing the decomposition (25) in (24) and using the relations (27)-(29), we obtain a nonlinear functional differential equation for the interacting part $W_{\text {int }}$ :

$$
\begin{aligned}
G_{13}^{-1} & \frac{\delta W_{\mathrm{int}}}{\delta G_{23}^{-1}}-\frac{g}{2} V_{1345}\left[2 G_{34} G_{52}-\left(G_{32} \frac{\delta W_{\mathrm{int}}}{\delta G_{45}^{-1}}+G_{54} \frac{\delta W_{\mathrm{int}}}{\delta G_{23}^{-1}}\right)+\frac{\delta^{2} W_{\mathrm{int}}}{\delta G_{23}^{-1} \delta G_{45}^{-1}}+\frac{\delta W_{\mathrm{int}}}{\delta G_{23}^{-1}} \frac{\delta W_{\mathrm{int}}}{\delta G_{45}^{-1}}\right] \\
& -e^{2} F_{\overline{12} 13}\left[\frac{1}{2} D_{\overline{21}} G_{32}-\left(\frac{1}{2} D_{\overline{21}} \frac{\delta W_{\mathrm{int}}}{\delta G_{23}^{-1}}+G_{32} \frac{\delta W_{\mathrm{int}}}{\delta D_{\overline{12}}^{-1}}\right)+\frac{\delta^{2} W_{\mathrm{int}}}{\delta D_{\overline{12}}^{-1} \delta G_{23}^{-1}}+\frac{\delta W_{\mathrm{int}}}{\delta D_{\overline{12}}^{-1}} \frac{\delta W_{\mathrm{int}}}{\delta G_{23}^{-1}}\right] \\
& +e^{2} H_{\overline{1} 13} D_{\overline{12}} H_{\overline{2} 45}\left[G_{52} G_{34}+G_{32} G_{54}-\left(G_{32} \frac{\delta W_{\mathrm{int}}}{\delta G_{45}^{-1}}+G_{54} \frac{\delta W_{\mathrm{int}}}{\delta G_{23}^{-1}}\right)+\frac{\delta^{2} W_{\mathrm{int}}}{\delta G_{23}^{-1} \delta G_{45}^{-1}}+\frac{\delta W_{\mathrm{int}}}{\delta G_{23}^{-1}} \frac{\delta W_{\mathrm{int}}}{\delta G_{45}^{-1}}\right] \\
& +e^{2} H_{\overline{1} 13} D_{\overline{12}} F_{\overline{23} 45}\left[-G_{32} \frac{\delta W_{\mathrm{int}}}{\delta H_{\overline{3} 45}}+\frac{\delta^{2} W_{\mathrm{int}}}{\delta G_{23}^{-1} \delta H_{\overline{3} 45}}+\frac{\delta W_{\mathrm{int}}}{\delta G_{23}^{-1}} \frac{\delta W_{\mathrm{int}}}{\delta H_{\overline{3} 45}}\right]=0 .
\end{aligned}
$$

With the help of Eqs. (9) and (13), this equation becomes

$$
\begin{aligned}
& -G_{12} \frac{\delta W_{\mathrm{int}}}{\delta G_{12}}-\frac{g}{2} V_{1234}\left[2 G_{23} G_{41}+4 G_{21} G_{53} G_{46} \frac{\delta W_{\mathrm{int}}}{\delta G_{56}}+G_{51} G_{26} G_{73} G_{48}\left(\frac{\delta^{2} W_{\mathrm{int}}}{\delta G_{56} \delta G_{78}}+\frac{\delta W_{\mathrm{int}}}{\delta G_{56}} \frac{\delta W_{\mathrm{int}}}{\delta G_{78}}\right)\right] \\
& -e^{2} F_{\overline{12} 12}\left[\frac{1}{2} D_{\overline{21}} G_{21}+\frac{1}{2} D_{\overline{21}} G_{31} G_{24} \frac{\delta W_{\mathrm{int}}}{\delta G_{34}}+G_{21} D_{\overline{31}} D_{\overline{24}} \frac{\delta W_{\mathrm{int}}}{\delta D_{\overline{34}}}+D_{\overline{31}} D_{\overline{24}} G_{31} G_{24}\left(\frac{\delta W_{\mathrm{int}}}{\delta D_{\overline{34}} \delta G_{34}}+\frac{\delta W_{\mathrm{int}}}{\delta D_{\overline{34}}} \frac{\delta W_{\mathrm{int}}}{\delta G_{34}}\right)\right] \\
& +e^{2} H_{\overline{1} 12} D_{\overline{12}} H_{\overline{2} 34}\left[G_{41} G_{23}+G_{21} G_{43}+2\left(G_{21} G_{53}+G_{51} G_{23}\right) G_{46} \frac{\delta W_{\mathrm{int}}}{\delta G_{56}}+G_{51} G_{26} G_{73} G_{48}\left(\frac{\delta^{2} W_{\mathrm{int}}}{\delta G_{56} \delta G_{78}}\right.\right. \\
& \left.\left.+\frac{\delta W_{\mathrm{int}}}{\delta G_{56}} \frac{\delta W_{\mathrm{int}}}{\delta G_{78}}\right)\right]-e^{2} H_{\overline{1} 12} D_{\overline{12}} F_{\overline{23} 34}\left[G_{21} \frac{\delta W_{\mathrm{int}}}{\delta H_{\overline{3} 34}}+G_{51} G_{26}\left(\frac{\delta^{2} W_{\mathrm{int}}}{\delta G_{56} \delta H_{\overline{3} 34}}+\frac{\delta W_{\mathrm{int}}}{\delta G_{56}} \frac{\delta W_{\mathrm{int}}}{\delta H_{\overline{3} 34}}\right)\right]=0 .
\end{aligned}
$$

From this functional differential equation, a graphical recursion relation can be derived. This is the subject of the next section.

\section{GRAPHICAL RECURSION RELATION}

From (31), we can derive a graphical recursion relation for the connected vacuum diagrams. When considering the loop expansion of the interaction part $W_{\mathrm{int}}$, one term is of one loop number larger than the other terms, as we now show.

The operators $G_{12} \delta / \delta G_{12}$ and $D_{\overline{12}} \delta / \delta D_{\overline{12}}$ simply count the number of scalar lines $I_{\mathrm{q}}$ and photon lines $I_{\gamma}$, respectively, in a given diagram. These numbers can be extracted from the number and type of vertices. Denoting by $V_{g}, V_{e}$ and $V_{e^{2}}$ the number of $g, e$ and $e^{2}$ vertices, we have the following counting rules. The Yukawa vertex $H_{\overline{1} 12}$ has one photon line and two scalar lines, while the quartic photon-scalar vertex $F_{\overline{12}}{ }_{2}$ has two photon lines and two scalar lines. Furthermore two vertex lines are necessary to produce an internal line when combining vertices. We have the obvious relation $2 I_{\gamma}=2 V_{e^{2}}+V_{e}$. Taking the quartic scalar self-interaction $V_{1234}$ into account, we have also $2 I_{\mathrm{q}}=2 V_{e^{2}}+2 V_{e}+4 V_{g}$. An odd number of photon fields gives no contribution to the free energy. Thus the vertex $e$ enters with even power. The number of loops is then easily found to be $L-1=V_{g}+V_{e} / 2+V_{e^{2}}$. Together, we have the counting rules 


$$
\begin{aligned}
I_{\gamma} & =V_{e^{2}}+\frac{V_{e}}{2}, \\
I_{\mathrm{q}} & =V_{e^{2}}+V_{e}+2 V_{g}, \\
L-1 & =V_{e^{2}}+\frac{V_{e}}{2}+V_{g} .
\end{aligned}
$$

These relations can be inverted to give the number of each type of vertex as a function of the number of loops, scalar, and photon internal lines:

$$
\begin{aligned}
V_{e^{2}} & =2(L-1)-I_{\mathrm{q}}, \\
\frac{V_{e}}{2} & =I_{\mathrm{q}}+I_{\gamma}-2(L-1), \\
V_{g} & =L-1-I_{\gamma} .
\end{aligned}
$$

It is now clear that, to count the number of scalars in a given diagram, it is only necessary to know the loop order, as well as the number of the quartic $e^{2}$ photon-scalar seagull vertices:

$$
I_{\mathrm{q}}=2(L-1)-V_{e^{2}} .
$$

The vertices $V_{e}$ are not taken into account when counting the quartic vertices $V_{e^{2}}$, which, by definition, count the two photon-two scalar vertices only. The relation (38) is interesting, since, for complicated diagrams, with a large loop-order, it is much less involved to count the number of quartic $e^{2}$ photon-scalar seagull vertices than the number of scalar lines.

We are now ready to demonstrate that equation (31) allows for a recursive solution. We form the loop expansion

$$
W_{\mathrm{int}}=\sum_{L=2}^{\infty} g^{L-1} W^{(L)}
$$

supposing that the vertices $e$ and $e^{2}$ are of order $\sqrt{g}$ and $g$, respectively. This is because the relevant parameter for the loop expansion is the inverse temperature $\beta=1 /\left(k_{B} T\right)$ which appears as a coefficient in front of the energy. The inverse temperature is set equal to one in this work (see Eq. (ii)). We could have restored it to show the loop counting. We would have seen readily that this would have been equivalent to the statement that $e$ and $e^{2}$ are of order $\sqrt{g}$ and $g$, respectively. In Eq. (39), $W^{(L)}$ is a sum over the different diagrams of a given order $L$ :

$$
W^{(L)}=\sum_{d}(-1)^{V_{g}+V_{e^{2}}} W^{(L, d)},
$$

where $d$ distinguishes between different classes of diagrams of the same loop order. The factor $(-1)^{V_{g}+V_{e^{2}}}$ takes care of minus signs in the diagrams of the perturbation expansion. Since the number of Yukawa vertices is even, it does not enter this prefactor: $(-1)^{V_{e}}=1$. Applying the scalar number operator on $W_{\text {int }}$ gives

$$
G_{12} \frac{\delta W_{\text {int }}}{\delta G_{12}}=\sum_{L=2}^{\infty} g^{L-1} \sum_{d}(-1)^{V_{g}+V_{e^{2}}} I_{\mathrm{q}}(L, d) W^{(L, d)},
$$

which stresses that each class of diagrams, i.e., each topology, has its own scalar number.

By performing the loop expansion (39), the contributions $W^{(L)}$ to the negative free energy consists of all connected vacuum diagrams constructed according to the following Feynman rules. A straight line and a wiggly line represent the free correlation of the scalar and the photon field, respectively:

$$
\begin{aligned}
1 \multimap 2 & \equiv G_{12}, \\
\overline{1} \sim \overline{2} & \equiv D_{\overline{12}} .
\end{aligned}
$$

The vertices are correspondingly pictured by

$$
\begin{aligned}
& \text { 3. } \quad \equiv-e \int_{\overline{1} 23} H_{\overline{1} 23}, \\
& \times-e^{2} \int_{\overline{12} 34} F_{\overline{12} 34}, \\
& \times \quad \equiv-g \int_{1234} V_{1234} .
\end{aligned}
$$


With these Feynman rules, the insertion of the loop expansion (39) into (31) leads to the following equation

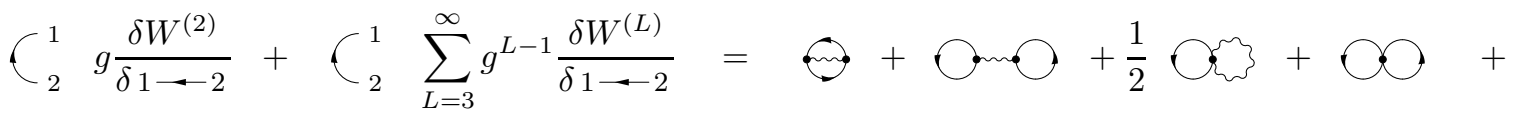

$$
\begin{aligned}
& \sum_{L=2}^{\infty} g^{L-1}\left[2 \bigcirc{ }_{2}^{1} \frac{\delta W^{(L)}}{\delta 1 \multimap-2}+\frac{1}{2} \leftarrow_{3}^{1} \frac{\delta^{2} W^{(L)}}{\delta 1 \multimap-2 \delta 3 \multimap 4}+\frac{1}{2}\right.
\end{aligned}
$$

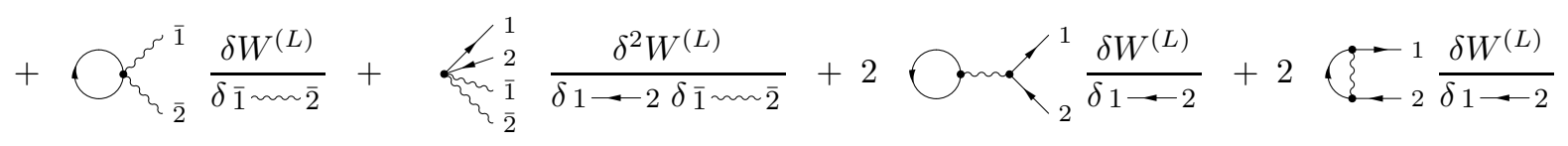

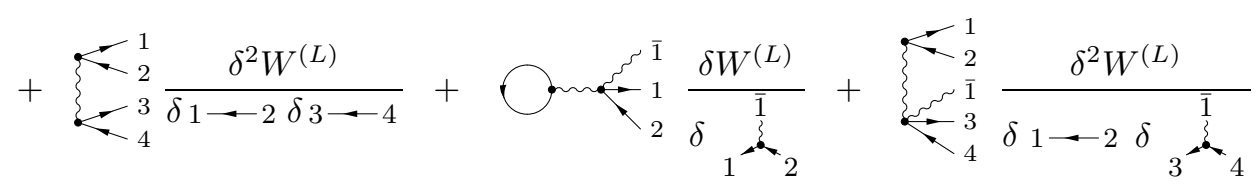

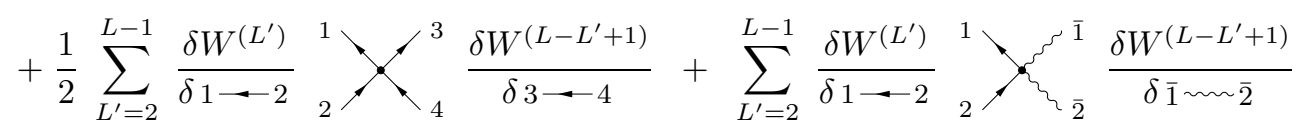

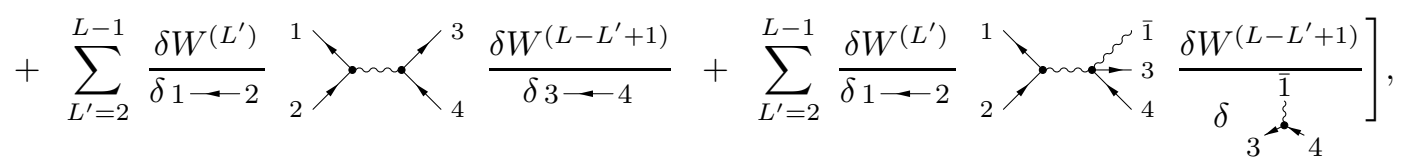

which we have written directly as a graphical equation where the vertices already contain the coupling constants $e, e^{2}, g$. The last four terms are the nonlinear ones. They start to give a contribution only at the three-loop level. (Although the overall summation starts at $L^{\prime}=2$, the nonlinear terms have an internal summation for $L^{\prime} \in[2, L-1]$, leading to a vanishing two-loop contribution.) The fact that only positive signs enter is a consequence of the Feynman rules (44)-(45).

The term $W^{(2)}$, corresponding to two-loop diagrams, is the initial condition to enter this equation. It needs not to be given because it is also contained in (47). Identifying the terms of order $g$, we see directly that only the first line survives. Integrating the equation gives the four diagrams in the right-hand-side, with the corresponding weights $1 / 2,1 / 2,1 / 2,1 / 2$ obtained by dividing each graph by its number of scalar lines (compare Table I):

$$
W^{(2)}=\frac{1}{2} \Theta+\frac{1}{2} \bigcirc \mathrm{Om}+\frac{1}{2} \mathrm{O}^{3}+\frac{1}{2} \bigcirc
$$

Equating powers of $g$, we end up with the following nonlinear graphical recursion relation for the vacuum diagrams:

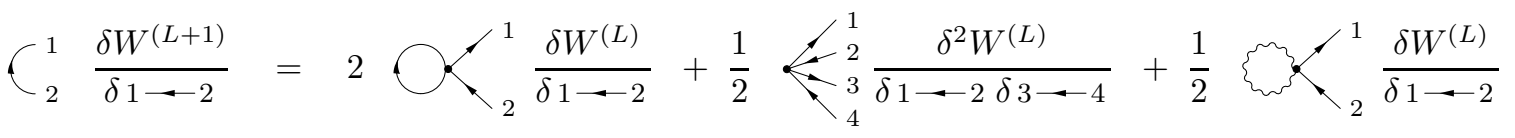

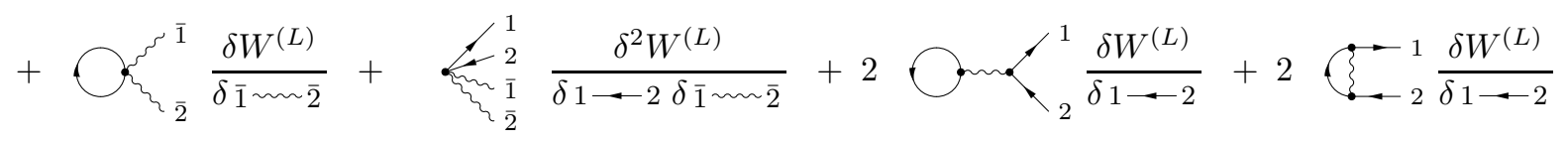

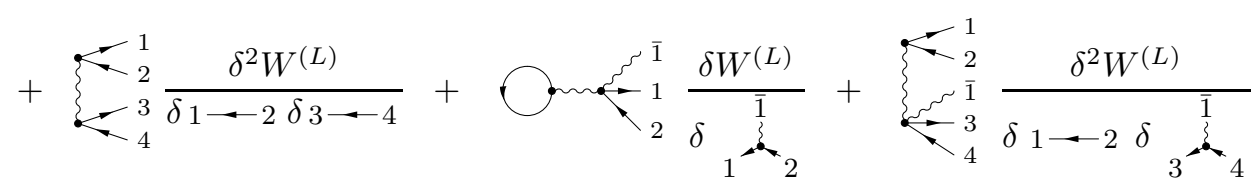




$$
\begin{aligned}
& +\frac{1}{2} \sum_{L^{\prime}=2}^{L-1} \frac{\delta W^{\left(L^{\prime}\right)}}{\delta 1 \longleftarrow 2} \nearrow_{2}^{1} \frac{\delta W^{\left(L-L^{\prime}+1\right)}}{\delta 3 \longleftarrow 4}+\sum_{L^{\prime}=2}^{L-1} \frac{\delta W^{\left(L^{\prime}\right)}}{\delta 1 \longleftarrow 2} \nearrow_{2}^{1} \frac{\delta W^{\left(L-L^{\prime}+1\right)}}{\delta \overline{1} m \overline{2}}
\end{aligned}
$$

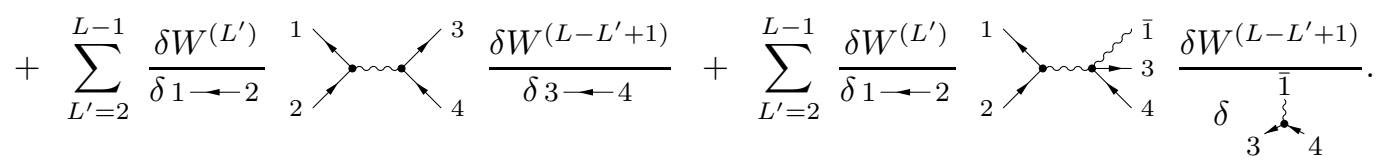

The recursive nature of this equation is due to the fact that the left-hand-side is of one higher loop order than the right-hand-side: To obtain the diagrams at order $L+1$, one applies the right-hand-side operators on the lower order diagrams. Since the left-hand-side contains the scalar number operator for each diagram, the weight of the diagram which have just been obtained has to be divided by the respective number of scalar lines, see Eq. (41). We remind the reader that this number can be obtained knowing the number of seagull vertices of the respective diagram, see Eq. (38).

We have derived the vacuum diagrams of the Ginzburg-Landau theory up to four loops which involves the nonlinear part of Eq. (49). Indeed, the nonlinear terms only enter from the three-loop order. The resulting diagrams are given in Table 1. The effort needed to obtain them is considerably reduced compared to a calculation done with the help of external sources coupled linearily to the fields. Note that Eq. (49) is suitable for an automatized symbolic computation which can be implemented as in Ref. [3], such that one may proceed to higher orders without much effort except for computer time.

A look at Table 1 shows that the diagrams and weights of the Yukawa part coincide with those from Ref. [4], as it should. For the pure $\phi^{4}$-part, the diagrams are equivalent to those in Ref. [3], although the weights do not coincide, since we deal with complex scalar fields.

Being in the possession of all Feynman diagrams we must still calculate the associated integrals in order to extract physical results. This will be done in a separate publication. In particular, we intend to compute the vacuum energy which determines the critical behavior of the heat capacity of a superconductor at the phase transition.

\section{CONCLUSIONS}

In this paper, we set up a graphical recursion relation for obtaining the connected diagrams of the Ginzburg-Landau model which describes superconductors near the critical point. We have used our equation to obtain the diagrams up to the four-loop order. These diagrams will be needed to extend our two-loop calculations in Ref. [1] to higher orders.

\section{ACKNOWLEDGEMENT}

The work of B.V.d.B. was supported by the Alexander von Humboldt foundation and the Institut Interuniversitaire des Sciences Nucléaires de Belgique.

[1] H. Kleinert and B. Van den Bossche, Two-loop effective potential of $O(N)$-symmetric scalar QED in $4-\epsilon$ dimensions; eprint: cond-mat/0104102.

[2] S. Schelstraete and H. Verschelde, Z. Phys. C 67, 347 (1995).

[3] H. Kleinert, A. Pelster, B. Kastening, and M. Bachmann, Phys. Rev. E 62, 1537 (2000); eprint: hep-th/9907168.

[4] M. Bachmann, H. Kleinert, and A. Pelster, Phys. Rev. D 61, 085017 (2000); eprint: hep-th/9907044.

[5] B. Kastening, Phys. Rev. E 61, 3501 (2000); eprint: hep-th/9908172.

[6] H. Kleinert and A. Pelster, Functional differential equations for the free energy and the effective energy in the brokensymmetry phase of $\phi^{4}$-theory and their recursive graphical solution; eprint: hep-th/0006153.

[7] H. Kleinert, Fortschr. Phys. 30, 187 (1982); H. Kleinert, Fortschr. Phys. 30, 351 (1982).

[8] A.N. Vasiliev, Functional Methods in Quantum Field Theory and Statistical Physics (Gordon and Breach Science Publishers, New York, 1998); translation from the Russian edition (St. Petersburg University Press, St. Petersburg, 1976). 


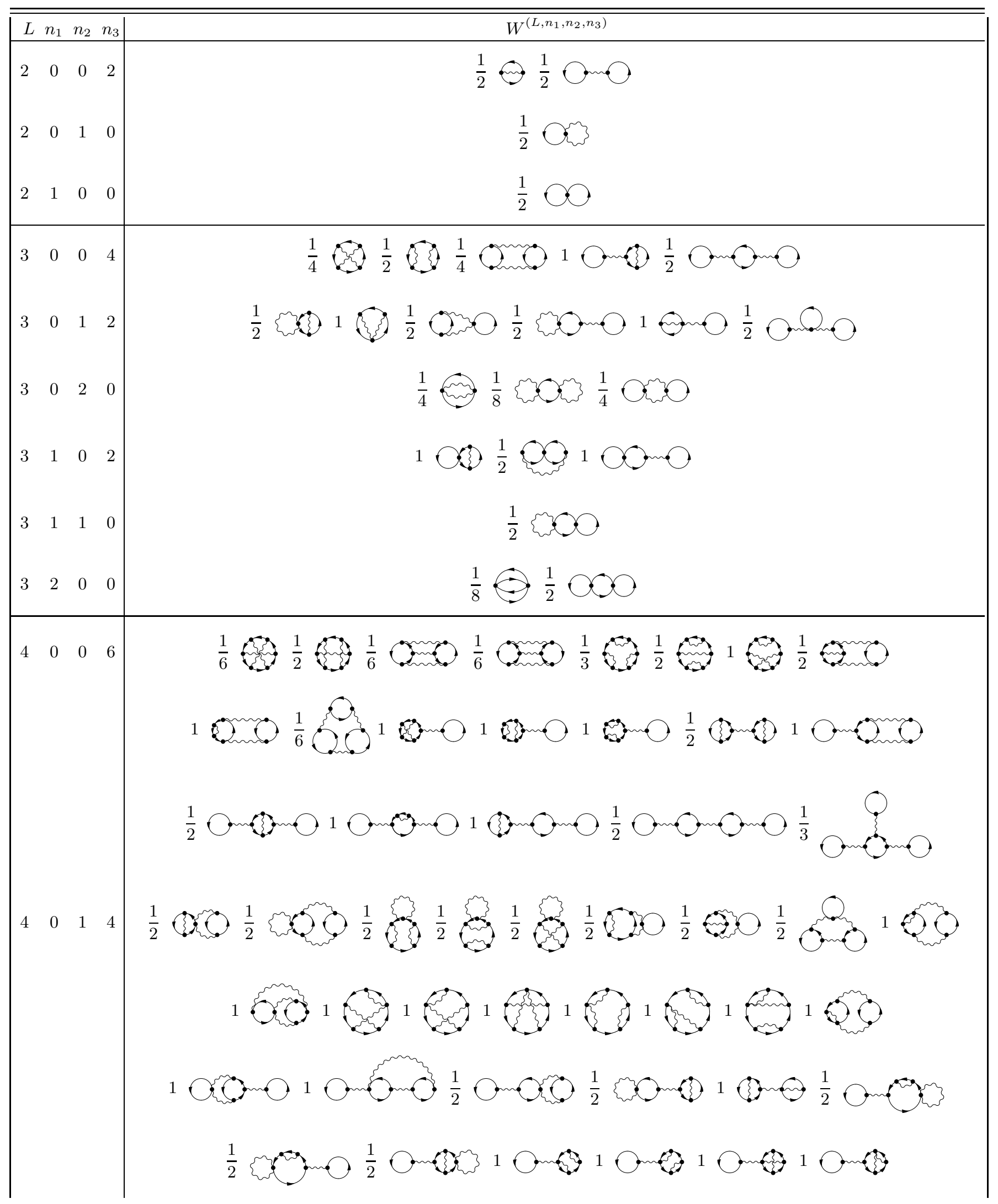




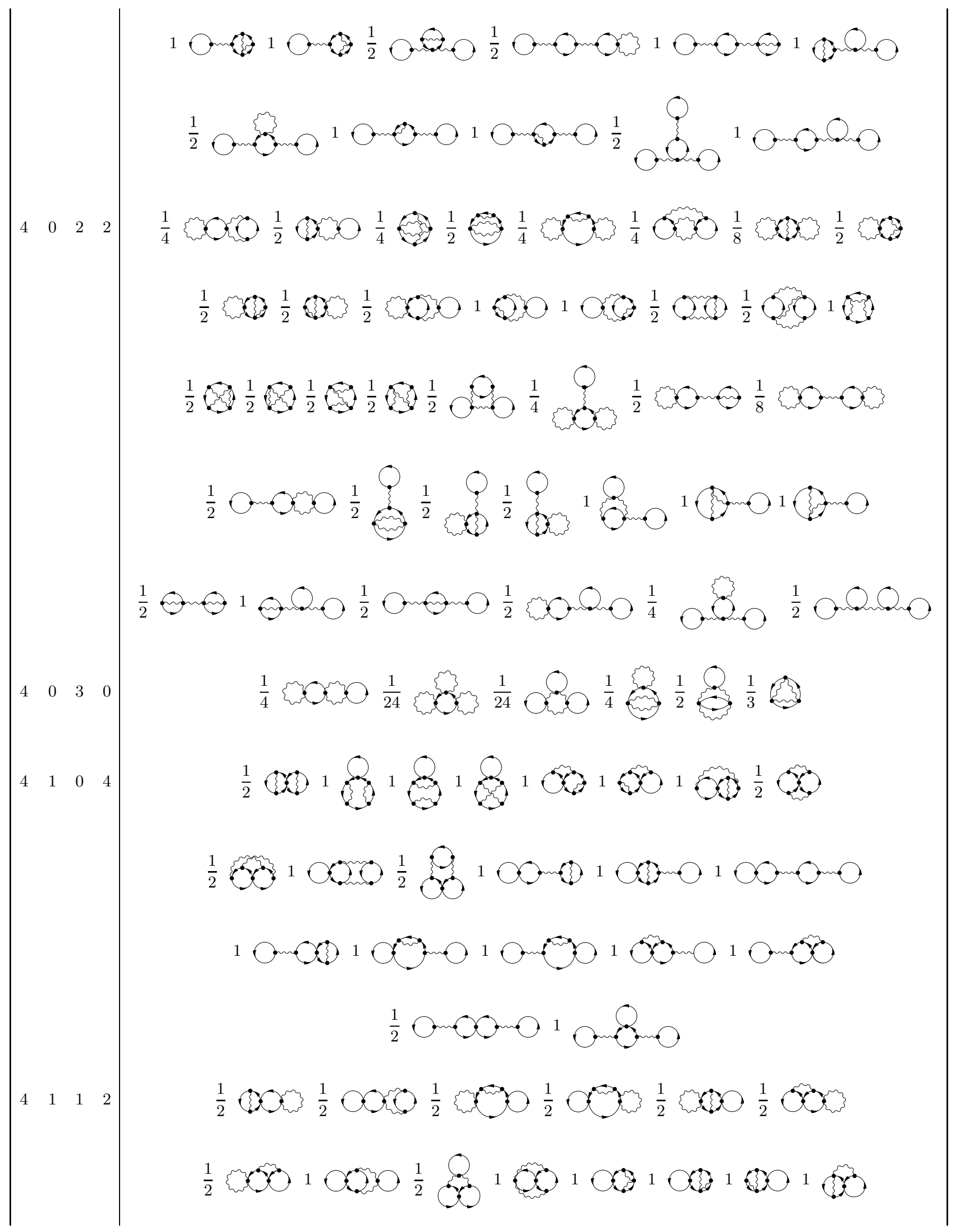




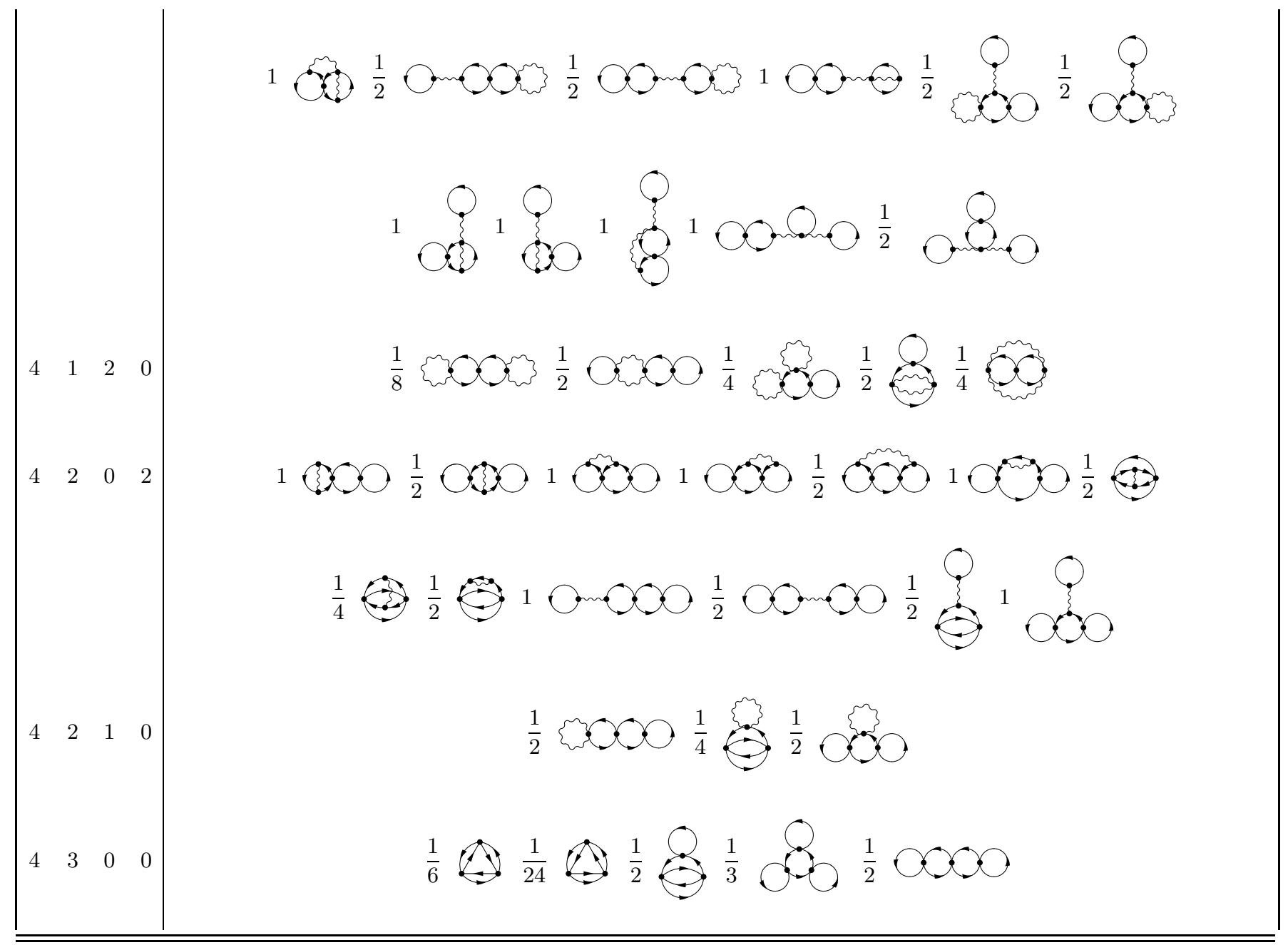

TABLE I. Connected vacuum diagrams $W^{\left(L, n_{1}, n_{2}, n_{3}\right)}$ and their weights up to the four-loop order of the $O(2)$ Ginzburg-Landau model, wehre $L$ denotes the loop order and $n_{1}, n_{2}, n_{3}$ count the number of vertices $V, F, H$, respectively. 